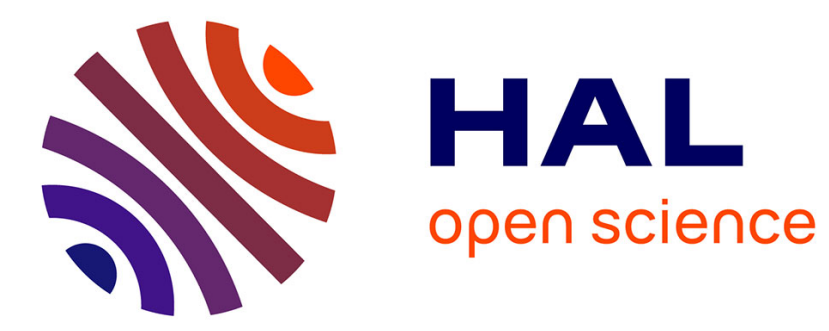

\title{
Interference model and antenna parameters setting effects on 4G-LTE networks coverage
}

Nourredine Tabia, Alexandre Gondran, Oumaya Baala, Alexandre Caminada

\section{To cite this version:}

Nourredine Tabia, Alexandre Gondran, Oumaya Baala, Alexandre Caminada. Interference model and antenna parameters setting effects on 4G-LTE networks coverage. PM2HW2N 2012, 7th ACM workshop on Performance Monitoring and Measurement of Heterogeneous Wireless and Wired Networks, Oct 2012, Paphos, Cyprus. pp 175-182, 10.1145/2387191.2387215 . hal-00938863

\section{HAL Id: hal-00938863 \\ https://hal-enac.archives-ouvertes.fr/hal-00938863}

Submitted on 16 May 2014

HAL is a multi-disciplinary open access archive for the deposit and dissemination of scientific research documents, whether they are published or not. The documents may come from teaching and research institutions in France or abroad, or from public or private research centers.
L'archive ouverte pluridisciplinaire HAL, est destinée au dépôt et à la diffusion de documents scientifiques de niveau recherche, publiés ou non, émanant des établissements d'enseignement et de recherche français ou étrangers, des laboratoires publics ou privés. 


\section{Interference Model and Antenna Parameters Setting Effects on 4G-LTE Networks Coverage}

\author{
Nourredine Tabia \\ University of Technology Belfort-Montbéliard \\ 90000, Belfort, France \\ nourredine.tabia@utbm.fr \\ Oumaya Baala \\ University of Technology Belfort-Montbéliard \\ 90000, Belfort, France \\ oumaya.baala@utbm.fr
}

\author{
Alexandre Gondran \\ Ecole Nationale d'Aviation Civile, ENAC \\ 31055, Toulouse, France \\ alexandre.gondran@enac.fr
}

\author{
Alexandre Caminada \\ University of Technology Belfort-Montbéliard \\ 90000, Belfort, France \\ alexandre.caminada@utbm.fr
}

\begin{abstract}
The currently emerging Long Term Evolution 4G-LTE cellular networks are based on new technique of transmission called the Orthogonal Frequency Division Multiple Access (OFDMA). This paper shows the interest of robust approach due to the uncertainty of traffic distribution. First, we develop and validate the interference model based on SINR metric for the deployment of the LTE network, and then we use greedy algorithms to show how frequency and tilt parameter settings can impact the coverage performance metric. Two frequency schemes have been compared to validate our model: the frequency reuse 1 scheme whereby the whole available bandwidth is used in each cell/sector and the frequency reuse 3 scheme in which the entire bandwidth is divided into 3 non-overlapping groups and assigned to 3 co-site sectors within each cell.
\end{abstract}

\section{Categories and Subject Descriptors}

C.2.1 [Computer-Communication networks]: Wireless communication; I.6.5 [Simulation and Modeling]: Model development- modeling methodologies

\section{General Terms}

Algorithms, Management, Measurement, Documentation, Performance, Design, Experimentation.

\section{Keywords}

LTE; SINR; Interference; frequency optimization; tilt optimization; robustness.

\section{INTRODUCTION}

Looking at the recent development of mobile radio access networks, it appears that size and complexity of cellular networks is growing at breakneck speed.

process parameters becomes more complicated and more expensive. Orthogonal Frequency Division multiplexing (OFDM)

Permission to make digital or hard copies of all or part of this work for personal or classroom use is granted without fee provided that copies are not made or distributed for profit or commercial advantage and that copies bear this notice and the full citation on the first page. To copy otherwise, or republish, to post on servers or to redistribute to lists, requires prior specific permission and/or a fee.

$P M^{2} H W^{2} N^{\prime} 12$, October 21-22, 2012, Paphos, Cyprus.

Copyright 2012 ACM 978-1-4503-1626-2/12/10...\$15.00. has been widely accepted as a promising air-interface technology for future generation systems by various standardization bodies and forums, for example, the third Generation Partnership Project (3GPP LTE), worldwide interoperability for microwave access (WIMAX), and the wireless world initiative new radio (WINNER) [3]. These techniques provide orthogonality between the users, reduce interference and improve the network Quality of Service (QoS). In addition, to make the network design process time-efficient, Self-Organizing Network (SON) functionalities added within LTE architecture by incorporating automated optimization, can significantly reduce deployment and maintenance costs (CAPEX and OPEX for CAPital EXpenditure and OPerating EXpenditure respectively). In a wireless network with several cells, the main key to improve the achievable average throughput by user is to mitigate the inter-cell interference caused by using the same frequency within adjacent cells. Various frequency reuse schemes have been proposed in literature $[1,2]$. Mitigation technique is generally categorized into three major techniques as shown in [3]: interference cancellation, interference averaging and interference avoidance techniques. The focus in this paper is on avoidance through inter-cell coordination, to achieve better network performance goals [3, 4, 5]. Resource allocation in radio networks essentially depends on the quality of some reference signals received by the User Equipment (UE). In LTE, they are the Reference Signal Received Power (RSRP) and the Reference Signal Received Quality (RSRQ) corresponding respectively to Received Signal Code Power (RSCP) and Ec/No in UMTS. Each user is assigned a portion of the spectrum depending on RSRP and RSRQ. The more complex optimization of reference signal is the RSRQ which is based on Signal to Interference plus Noise Ratio (SINR) $[2,6]$. SINR is an important performance indicator for estimating the achievable throughput, taking into account the interference received from the neighboring cluster of first-tier cells. The estimation and optimization of the SINR are well-known problems in radio communication systems such as 802.11, GSM or UMTS $[11,12,13]$, and LTE needs also a good estimation and control of SINR.

Optimizing antenna parameters configuration to meet variant of service and performance requirement is one of main targets of next generation networks. It can significantly improve the coverage and the capacity of the network dealing with the lack of available bandwidth in base stations. Several studies have been done in this direction to understand the impact of parameters on antennal quality of service offered by the network $[6,7,14]$. E. 
Dinan and A. Kurochkin have measured [14] the impact of azimuth and tilt inaccuracies on network performance considering three main quality parameters: service coverage, soft handover areas and the ratio of chip energy to interference Ec/No. The optimization tool used to improve the group of KPIs (Key Performance Indicators) cited above is the recursive optimization algorithm [14]. Many simulation results show that azimuth error in the range of \pm 8 degrees is tolerable to improve the performance of 42 sites network scenario. Tilt parameter has the same effect on Network performance while setting tolerance is just about \pm 0.5 degrees. The approach of simulated annealing is used in [7] to study number of network configuration parameters (CPICH power, mechanical downtilt, electrical downtilt and antenna azimuth) effects toward coverage service in UMTS network. The case study demonstrates how the automated optimization can offer a significant performance gain like power consumption of common channels which is a strong indication of reducing the interference in the network. In modern communication systems, further parameters investigations have been developed to meet requirement set. Various combinations of antenna have been studied in term of SINR and throughput performance in LTE case, outlined for example in $[6,15]$.

This paper is organized in two main parts: in the first part, we evaluate the Interference model based on SINR for both reuse 1 and 3. The SINR will include a new parameter compared to the classical model. Our contribution on this part consists on adding the load factor of cells to measure the impact of the traffic on the interference model. In the second part, we study the influence of the frequency and the tilt parameters on some performance metrics (quality of the SINR, users in outage...) and also emphasize the interest of robust optimization considering both frequency and tilt as important design parameters when tuning live network. The choice of the robust approach is mainly due to the uncertainty of the traffic distribution due to the traffic change. For this aim, the paper is structured as follows. Section 2 introduces the system model and basic assumptions. Section 3 extends this work and shows the performance metrics and test assumptions. Subsequently, section 4 describes the algorithms and section 5 presents some results to highlight the use of SINR interference model and shows the utility of robust optimization toward the change on the traffic demand. Conclusion and perspectives are drawn in section 6 .

\section{SYSTEM MODEL}

\subsection{Basic Assumptions}

In this paper we consider the downlink transmission and illustrate the interference schemes using a theoretical model of seven-cell hexagonal layout as shown in Figure 1.

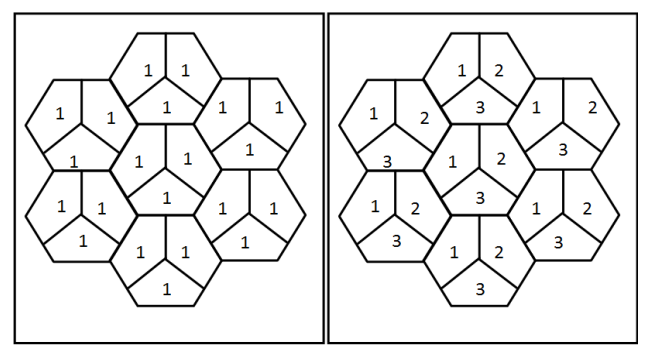

a)

b)

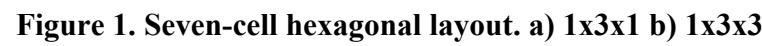

Three sectors are considered in each site with three base stations. In (a) we see the frequency reuse $1 \times 3 \times 1$ pattern where 1 site with 3 sectors use 1 frequency set, that is the same frequency set for all sectors. In (b) we see the frequency reuse $1 \times 3 \times 3$ pattern where 1 site with 3 sectors use 3 frequency sets, that is different frequency set for all sectors. The $1 \times 3 \times 3$ requires a frequency plan but should avoid interference when the network load becomes too high to apply the $1 \times 3 \times 1$ pattern. The features of our computational model are the following:

1. Intra-frequency interference is avoided due to the use of OFDMA technique in downlink transmission. In LTE the orthogonality between subcarriers insures that the interference inside the cell can be ignored.

2. The basic resource element in OFDMA is the physical resource block $(\mathrm{PRB})$ which spans both frequency and time dimensions. In this paper, we do not take into account PRB to estimate the inter-cell interference; we only focus on the frequency sub-band reuse scheme. Two adjacent cells are scrambling each other if they are using the same sub-band to transmit data. It gives a worst but fast estimation of SINR.

3. Scenarios are used to represent the traffic distribution of our model since LTE network traffic is unpredictable now. The interference model defined here and based on SINR depends not only on antenna parameters (frequency, tilt, azimuth...) but also on traffic load along day; then in our computation the users available in target sector or adjacent sectors can affect the received signal from other users. As we consider that all users communicate at the same time, a scenario shows the distribution of demand at a given time.

4. We introduce a performance indicator to measure occupancy rate on each sector, the load factor. This indicator is a ratio between used bandwidth and the maximum available bandwidth. It is measured for each sector of the network. The feature of the load factor is to determine the bottlenecks of the network and the overloaded sectors. This indicator will be used in the interference model.

\subsection{Case Study and Problem Formulation}

The considered network for this study consists of tri-sectors sites in the city of Belfort, located in the north-eastern of France. It is a $40 \mathrm{~km} \times 20 \mathrm{~km}$ area with a lot of big industrial companies and the population is about 200,000 people, that includes inhabitants and people coming from outside the area for work. For our model the service area is divided into a grid of equally sized test points. A test point is a $25 \times 25$ meters area.

Due to the very small size of the test point, we assume the same signal propagation conditions within a test point; it means that all users located inside a test point have the same signal and the same RSRP, SINR and RSRQ. Thus, a test point determines the resolution of the computation and the amount of data on signals. A test point is characterized by its number of users and the category of required services for each user (voice, data...). Each sector in the network is equipped with one directive antenna. Each antenna is characterized by its parameters: radiation pattern, azimuth, electrical and mechanical tilts, gain in transmission and reception, frequency and output power in downlink. Due to the dynamic aspect of the network and changes in traffic demand, we introduce the concept of traffic scenarios. A scenario is a given distribution and load of the traffic demand at a given time for each test point of the map. Then, several scenarios allow us to 
compute different situations of network performance to study the robustness problem.

The problem formulation is given by the following sets of data, parameters and functions.

Let: $\mathrm{B}=\left\{1, \ldots, \mathrm{n}^{\mathrm{B}}\right\}$, the set of $n^{B}$ base stations eNB of the network; $\mathrm{T}=\left\{1, \ldots, \mathrm{n}^{\mathrm{TP}}\right\}$, the set of $n^{T P}$ test points of the map; and $C_{t, s}$, the number of users located on the test point $t$ in scenario $s$.

The interference model based on SINR is thus calculated as defined in Equation 1:

$$
\gamma_{\mathrm{b}, \mathrm{t}, \mathrm{s}}=\frac{\mathrm{p}_{\mathrm{b}, \mathrm{t}}^{\mathrm{R}} \mathrm{f}_{\mathrm{b}}}{\sum_{\mathrm{b}^{\prime} \neq \mathrm{b}, \mathrm{f}_{\mathrm{b}}=\mathrm{f}_{\mathrm{b}^{\prime}}} \mathrm{p}_{\mathrm{b}^{\prime}, \mathrm{t}}^{\mathrm{R}} \mathrm{f}_{\mathrm{b}^{\prime}} \delta_{\mathrm{b}, \mathrm{s}} \delta_{\mathrm{b}^{\prime}, \mathrm{s}}+\mathrm{n}_{0} \mathrm{w}}
$$

Where, $\gamma_{\mathrm{b}, \mathrm{t}, \mathrm{s}}$ is the SINR received by the test point $t$ and issued from the eNB $b$ in scenario $s ; \mathrm{f}_{\mathrm{b}}$ and $\mathrm{f}_{\mathrm{b}^{\prime}}$ are the frequencies used by eNB $b$ and $b$, respectively; $\delta_{\mathrm{b}, \mathrm{s}}$ and $\delta_{\mathrm{b}, \mathrm{s}^{\prime}}$ are load factors that corresponds to base stations $b$ and $b$ in scenario $s$ (load factors are multiplied because we assume that the interference is proportional to the number of users using the same frequency in the target and neighbors cells). Base station $b$ is said to be saturated in scenario $s$ if its load factor $\delta_{\mathrm{b}, \mathrm{s}}$ is equal to 1 . The term $w$ represents the total bandwidth used by $b$ and $n_{0}$ is the thermal noise over the bandwidth $w$. The terms $\mathrm{p}_{\mathrm{b}, \mathrm{t}}^{\mathrm{R}}$ and $\mathrm{p}_{\mathrm{b}^{\prime}, \mathrm{t}}^{\mathrm{R}}$ are the end power received by UE located in test point $t$ from respectively $b$ and $b$ '. The estimation of this power is given by the Hata propagation model in Equation 2:

$$
\begin{aligned}
\mathrm{p}_{\mathrm{b}, \mathrm{t}}^{\mathrm{R}}(\mathrm{dBm})= & \mathrm{p}_{\mathrm{b}}^{\mathrm{E}}-\mathrm{PL}_{\mathrm{b}, \mathrm{t}}+\mathrm{g}_{\mathrm{b}}^{\mathrm{MAX}}-\mathrm{a}_{\mathrm{b}}^{\mathrm{VER}}\left(\theta_{\mathrm{b}, \mathrm{t}}-\mathrm{t}_{\mathrm{b}}^{\mathrm{M}}-\mathrm{t}_{\mathrm{b}}^{\mathrm{E}}\right)- \\
& \mathrm{a}_{\mathrm{b}}^{\mathrm{HOR}}\left(\varphi_{\mathrm{b}, \mathrm{t}}-\mathrm{a}_{\mathrm{b}}\right)
\end{aligned}
$$

Where, $\mathrm{p}_{\mathrm{b}}^{\mathrm{E}}$ is the power in $d B m$ issued from the eNB $b \cdot \mathrm{g}_{\mathrm{b}}^{\mathrm{MAX}}$ is the antenna gain while $\mathrm{a}_{\mathrm{b}}^{\mathrm{VER}}$ and $\mathrm{a}_{\mathrm{b}}^{\mathrm{HOR}}$ are the vertical and horizontal radiation pattern due to the position of the test point from the main beam of the antenna. As shown in Figure 2 (a), $a_{b}^{\text {VER }}$ depends essentially on the antenna tilt which is the angle of the main beam below the horizontal plane. We distinguish two different tilts: the mechanical tilt $\mathrm{t}_{\mathrm{b}}^{\mathrm{M}}$ to adjust the physical angle of the antenna brackets and the electrical tilt $t_{b}^{\mathrm{E}}$ which does not change the physical angle, but adjusts the radiating currents in the antenna elements to lower the beam in all horizontal directions. The right part of Figure 2 shows the impact of azimuth parameter on the horizontal radiation pattern. Th azimuth is the horizontal angle $a_{b}$ between the north and the antenna main lobe direction.

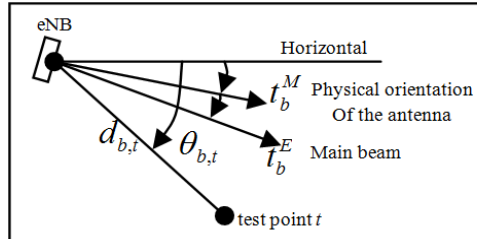

(a) Vertical cut

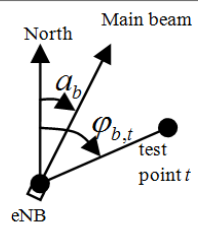

(b) Horizontal cut
Figure 2. Horizontal and vertical angles
In 3GPP LTE tests, we apply the two formulas [6] given by Equation 3 and Equation 4 for the computation of $\mathrm{a}_{\mathrm{b}}^{\text {VER }}\left(\theta_{\mathrm{b}, \mathrm{t}}, \mathrm{t}_{\mathrm{b}}^{\mathrm{M}}, \mathrm{t}_{\mathrm{b}}^{\mathrm{E}}\right)$ and $\mathrm{a}_{\mathrm{b}}^{\mathrm{HOR}}\left(\varphi_{\mathrm{b}, \mathrm{t}}, \mathrm{a}_{\mathrm{b}}\right)$ :

$\mathrm{a}_{\mathrm{b}}^{\mathrm{VER}}\left(\theta_{\mathrm{b}, \mathrm{t}}, \mathrm{t}_{\mathrm{b}}^{\mathrm{M}}, \mathrm{t}_{\mathrm{b}}^{\mathrm{E}}\right)=-\min \left[12\left(\frac{\theta_{\mathrm{b}, \mathrm{t}}-\mathrm{t}_{\mathrm{b}}^{\mathrm{M}}-\mathrm{t}_{\mathrm{b}}^{\mathrm{E}}}{\theta_{3 \mathrm{~dB}}}\right), \mathrm{SLA}_{\mathrm{v}}\right], \mathrm{SLA}_{\mathrm{v}}=20 \mathrm{~dB}$

$\mathrm{a}_{\mathrm{b}}^{\text {HOR }}\left(\varphi_{\mathrm{b}, \mathrm{t}}, \mathrm{a}_{\mathrm{b}}\right)=-\min \left[12\left(\frac{\varphi_{\mathrm{b}, \mathrm{t}}-\mathrm{a}_{\mathrm{b}}}{\varphi_{3 \mathrm{~dB}}}\right), \mathrm{A}_{\mathrm{m}}\right], \mathrm{A}_{\mathrm{m}}=25 \mathrm{~dB}$

Where, $S L A_{v}$ is the side lobe attenuation and $A_{m}$ is the front-toback attenuation [10]. $\theta_{3 d B}$ and $\Psi_{3 d B}$ are the half power beam width in vertical and horizontal plan respectively.

Finally, with the path loss the Hata model for urban areas is formulated as in Equation 5 [16]:

$$
\begin{aligned}
P L_{b, t}= & 69.55+26.16 \log \left(f_{0}\right)-13.82 \log \left(z_{b}\right)-a\left(z_{t}\right)+ \\
& \left(44.9-6.55 \log \left(z_{t}\right)\right) \log \left(d_{b, t}\right)
\end{aligned}
$$

And for small or medium sized city the value of $a\left(z_{t}\right)$ is:

$$
a\left(z_{t}\right)=0.8+\left(1.1 \times \log \left(f_{0}\right)-0.7\right) z_{t}-1.56 \log \left(f_{0}\right)
$$

Where, $P L_{b, t}$ is the path loss $(\mathrm{dB})$ in urban area between the eNB $b$ and the test point $t, f_{0}$ is the frequency (Mhz), $z_{b}$ and $z_{t}$ are the height of the base station $b$ and the test point $t(\mathrm{~m}), \mathrm{d}_{\mathrm{b}, \mathrm{t}}$ is the distance between the base station $b$ and the center of the test point $t(\mathrm{~m})$, while $a\left(z_{t}\right)$ is the correction factor for mobile unit antenna height (dB).

In the current work, we will consider two parameter settings of the antenna. The study focuses on the impact of the frequency and the tilt parameters on the number of non covered users in the service area. The robust approach uses the mean robustness over three different demand scenarios in a traffic day. The proposed evaluation methodology aims to show the effect of the antenna parameters on the coverage performance metric, with respect to traffic distribution. For the study presented in this paper, the formulation of the overall problem is done in the following.

\subsubsection{Decision variables (parameter settings)}

$t_{b}$ is the tilt orientation of the eNB $b: t_{b} \in T_{b}$

Where $T_{b}$ is the set of possible values of the tilt parameter.

$f_{b, n}$ is the variable for carrier assignment to eNB : $f_{b, n}=\{0,1\}$

$\mathrm{f}_{\mathrm{b}, \mathrm{n}}=\left\{\begin{array}{l}1 \text { if carrier } \mathrm{n} \text { is assigned to eNB } \mathrm{b} \\ 0 \text { otherwise }\end{array}\right.$

\subsubsection{Constraints}

The main constraints of our model are: (C1) $\forall b \in B, V_{b}^{M I N} \leq\left|V_{b}\right| \leq V_{b}^{M A X}$ : minimum and maximum number of neighborhood cells for $b$. $|\mathrm{Vb}|$ is the cardinal of neighborhood cells for $\mathrm{b}$.

(C2) $\forall t \in T, \sum_{b \in B} u_{b, t} \leq 1:$ a test point is associated with exactly one eNB. Where

$u_{b, t}=\left\{\begin{array}{l}1 \text { if test point } \mathrm{t} \text { is associated to the eNB } \mathrm{b} \\ 0 \text { Otherwise }\end{array}\right.$ 


$$
\forall b \in B, \sum_{n \in N} f_{b, n} \leq 1 \quad: \text { one eNB } b \text { can use only one }
$$

carrier $n$.

\subsubsection{Fitness function}

Let $\mathrm{n}_{0, \mathrm{~s}}^{\mathrm{C}}$ be the number of non covered users in scenario $s$.

$$
\mathrm{n}_{0, \mathrm{~s}}^{\mathrm{C}}=\sum_{\mathrm{t} \in \mathrm{T}_{0}^{\mathrm{C}}} \mathrm{n}_{\mathrm{t}, \mathrm{s}}^{\mathrm{C}}
$$

Where, $\mathrm{n}_{\mathrm{t}, \mathrm{s}}^{\mathrm{C}}$ is the number of non-covered users in test point $t$ for scenario $s$.

\subsubsection{Robustness function

$$
\mathrm{f}^{\mathrm{Rob}}=\sum_{\mathrm{s} \in \mathrm{S}} \mathrm{n}_{0, \mathrm{~s}}^{\mathrm{C}}
$$

Where, $\mathrm{f}^{\mathrm{Rob}}$ is the sum of non-covered users in all scenarios.

\section{TEST ASUMPTIONS AND PERFORMANCE METRICS}

The main parameters and assumptions we used are those selected by 3GPP for LTE as shown in Table 1. Evaluations are performed by a static snapshot of the network level.

Table1. Test assumptions for LTE downlink

\begin{tabular}{|c|c|}
\hline Parameters & Simulation setting \\
\hline Network layout & 35 sites and 88 sectors \\
\hline Required service/user & $2 \mathrm{Mbps}$ \\
\hline System frequency & $1800 \mathrm{Mhz}$ \\
\hline System bandwidth & $20 \mathrm{Mhz}$ \\
\hline Frequency reuse factor & $1 \times 3 \times 1$ and $1 \times 3 \times 3$ \\
\hline eNB heights range & {$[17 \mathrm{~m}, 46 \mathrm{~m}]$} \\
\hline UE height & $1.5 \mathrm{~m}$ \\
\hline Propagation loss model & Hata model $[16]$ \\
\hline TX power range & {$[39 \mathrm{dBm}, 46 \mathrm{dBm}]$} \\
\hline Mechanical tilt range & {$\left[0^{\circ}, 6^{\circ}\right]$} \\
\hline Electrical tilt range & {$\left[0^{\circ}, 10^{\circ}\right]$} \\
\hline Azimuth range & {$\left[0^{\circ}, 360^{\circ}\right]$} \\
\hline Horizontal HPBW & $+70^{\circ}$ \\
\hline Vertical HPBW & $+10^{\circ}$ \\
\hline Antenna gain range & {$[14 \mathrm{dBi}, 18.9 \mathrm{dBi}]$} \\
\hline Traffic distribution & Distribution in \\
& proportion to UMTS \\
& traffic load \\
\hline
\end{tabular}

In addition to table 1, further assumptions are used for robust optimization tests

1. Three realistic traffic scenarios are tested $(8 \mathrm{am}, 3 \mathrm{pm}$ and $6 \mathrm{pm})$

2. Frequency scheme reuse 3 is retained

3. Deterministic allocation of sub-bands as showed in the right part of Figure 1 is now used

4. The antennas are grouped by site and stored on the basis of an index in ascending order of the $\mathrm{x}$-axis

Various performance metrics are considered herein:

\subsection{Signal to Interference plus noise ratio}

The SINR, expressed in Equation 1, is an important indicator to evaluate cellular networks. SINR choice is motivated by the fact that:

1. It takes into account all the parameters of the antenna.

2. It depends on the traffic distribution and the load factor of the network.

3. It resizes the network and determines which base station controls each user.

4. It allows us to estimate the total throughput of the network.

\subsection{Load Factor}

The Load Factor of the sector/cell is the ratio between the total allocated bandwidth to the cell, that is the required bandwidth and the maximum total bandwidth available in the cell, that is the resources allocated to the cell. Let $\delta_{\mathrm{b}, \mathrm{s}}$ be the load factor, then: $\delta_{\mathrm{b}, \mathrm{s}}=\mathrm{w}_{\mathrm{b}, \mathrm{s}}^{\mathrm{S}} / \mathrm{w}$ where, $\mathrm{w}_{\mathrm{b}, \mathrm{s}}^{\mathrm{s}}$ is the total allocated bandwidth to the base station $b$ in the reference scenario $s$, and $w$ is its maximum available bandwidth. It is worthwhile to mention that load factor is one of the main key indicators in cellular networks. It has been suggested that the downlink cell load for a stable network should not exceed $70 \%$ [7]. Huge loaded cells are those for which $\delta_{\mathrm{b}, \mathrm{s}}>0.7$ and overloaded cells are those for which $\delta_{\mathrm{b}, \mathrm{s}}>1$.

\section{ALGORITHMS}

In order to show the interest of robust optimization, we present an algorithm able to quickly find a good solution (the greedy algorithm aims at improving the rate of non uncovered users on the total number of users dropped initially in the network). We were not looking for the best solution at this stage. An iterative algorithm is used; the purpose is not to find an optimal solution but to get the benefit of robust optimization for 3 scenarios in comparison with local solution based on a single scenario. The algorithm with several variants is proposed for each parameter: frequency allocation and tilt configuration. We measure the effect of each parameter toward the network coverage metric.

\subsection{Frequency parameter optimization}

It can be proved that frequency assignment problem is NP-hard as it is a graph-coloring problem. For such problems, guaranteeing optimum requires, in the worst case, an enumeration of all possible configurations. The number of possibilities is enormous; in our case study, for 35 cells, 88 antennas and 3 frequency groups, the number of possibilities is $6^{35}=1.71 * 10^{27}$. The robust optimization function takes into account the three scenarios considered above. The used algorithm is described in the following. We run the optimization with different varying conditions:

1. The scenarios of traffic: several traffic hours.

2. The procedure for the initial frequency assignment to the base stations: deterministic or stochastic per sector from the same site.

3. The sites neighborhood search to test the permutation of frequency: sites ranked from the input file or randomly chosen during optimization.

The algorithm starts with one solution using the reuse scheme $1 \times 3 \times 3$. The optimization algorithm is run for each scenario to show the best configuration of the frequency parameter setting with respect to the performance metric given by the Equation 6 . 
For each explored site, we evaluate the $6(3 !=6)$ possibilities of permutations for each sector of the site. The algorithm evaluates 6x88 permutations at each iteration. If a frequency permutation improves the evaluation function of the current solution, the algorithm keeps the last modification and goes through the next sector configuration. The algorithm stops once the current iteration brings no improvement. This is achieved by the following algorithm which was used for all cases.

\section{Algorithm for frequency optimization}

\section{Input parameters}

Set $B$ of $n^{B}$ base stations; Set $T$ of $n^{T P}$ test points; Set $S$ of scenarios:

$s_{1}=8 \mathrm{am}, s_{2}=3 \mathrm{pm}$ and $s_{3}=6 \mathrm{pm}$; Frequency reuse scheme $1 \times 3 \times 3$ (3 groups of frequency to assign to base station)

Variables

Frequency assignment to base stations

Fitness function

Fitness $(F)=$ Number of outage users for the frequency plan $F$

in $s$ for non robust optimization

in $s_{1}, s_{2}$ and $s_{3}$ for robust optimization

Algorithm:

Initialize $F / / F$ is the initial frequency plan

$F^{*}=F / / F^{*}$ is the current best frequency plan

Repeat

Improve $=$ False

For each site $b$ of the network // Testing all the sites

For each permutation $j=1: 6 / /$ Testing all the of frequency on $b$

Generate the new frequency plan $F$ from $F^{*}$

IF Fitness $(F)<$ Fitness $\left(F^{*}\right) / /$ Evaluation of the current configuration

$F^{*}=F / /$ Store the new best solution of frequency plan Improve $=$ True

$$
\text { End IF }
$$

End For

End For

Until Improve=False // Stopping criteria if there is no improvement.

\subsection{Tilt parameter optimization}

The same algorithm is implemented to optimize the tilt parameter of the antennas. Different configurations are tested using a discretization of the possible values. We aim at finding a good antenna down tilt orientation among a range of possible values $\left[0^{\circ}, 12^{\circ}\right]$ in degrees. For 35 cells and 88 antennas, we have $13^{88}=$ $1.06 * 10^{98}$ possibilities. It is impossible in such case to guarantee an optimum solution; we aim then at finding an acceptable solution and show the tilt effects on the traffic demand scenarios. We assume that

1. Three scenarios of traffic are considered

2. The starting solution on the tilt parameter is that given by the realistic GSM/UMTS network (operating data)

3. Sites in the neighborhood search are chosen at first by the order they are stored in the data file and then, randomly during optimization phase. So, we have two variants of the algorithm

We evaluate the 13 possible values for each antenna to meet better number of covered users, using the same starting solution and the same scenarios of traffic ( $8 \mathrm{am}, 3 \mathrm{pm}$, and $6 \mathrm{pm}$ ).

In the first variant of our algorithm, sites are processed in the order of storage in the data file, $13 * 88$ possible configurations are tested at each iteration. If the current tilt configuration improves the evaluation function of the current solution, we maintain the solution and then process the next neighborhood site chosen in the data file. The algorithm stops once the current iteration brings no improvement (stopping criteria). In the second variant we keep the same assumptions (same scenarios of traffic and same starting solution) but sites are processed randomly instead of the order in the data file. In this case, the stopping criteria considered here is the running time duration. The first variant of the algorithm is presented in the following

\section{Algorithm for tilt optimization}

Input parameters

Set B of nB base stations; Set T of nTP test points; Set S of scenarios:

$\mathrm{s} 1=8 \mathrm{am}, \mathrm{s} 2=3 \mathrm{pm}$ and $\mathrm{s} 3=6 \mathrm{pm}$; tilts in operation

$\underline{\text { Variables }}$

Tilt orientation of the base stations

Fitness function

Fitness $(T)=$ Number of outage users for the tilt configuration $T$

in $\mathrm{s}$ for non robust optimization

in $\mathrm{s} 1, \mathrm{~s} 2$ and $\mathrm{s} 3$ for robust optimization

Algorithm:

Initialize $\mathrm{T} / / \mathrm{T}$ is the initial Tilt configuration

$\mathrm{T}^{*}=\mathrm{T} / / \mathrm{T}^{*}$ is the current best frequency plan

Repeat

Improve $=$ False

For each antenna $\mathrm{b}$ of the network // Testing all the antenna

For each configuration $\mathrm{j}=0: 12 / /$ Testing all the tilt configurations on $\mathrm{b}$

Generate the new tilt configuration $\mathrm{T}$ from $\mathrm{T}^{*}$

IF Fitness(T)<Fitness( $\left.T^{*}\right) / /$ Evaluation of the current configuration

$\mathrm{T}^{*}=\mathrm{T} / /$ Store the new best solution of tilt configuration

Improve $=$ True

End IF

End For

End For

Until Improve $=$ False $/ /$ Stopping criteria if there is no improvement.

\section{RESULTS EVALUATION}

In order to evaluate the SINR model presented in section 2 , we focus on frequency reuse 1 ( $1 \times 3 \times 1$ pattern) and $3(1 \times 3 \times 3$ pattern). The baseline network used for our study is the city of Belfort described in section 2.2. The UE are randomly dropped in each cell in proportion to UMTS traffic load. We present in the following the methodology to evaluate the SINR model taking into account traffic data of the baseline network.

First, we assume that the base station is engaged to communicate with a UE if the SINR received by the UE is high enough, i.e. achieve the required SINR threshold to establish a communication. So, the UE is allocated to a base station according to the quality of the received SINR. We further assume that the load factor is considered here to calculate the SINR as mentioned in Equation 1. A cell is defined as a set of test points of the map; a test point is assigned to the base station which provides the best SINR. As a first step we assign the test point to the base station on the basis of the best RSRP and define the initial cell coverage for each station. Then we determine the number of UE per cell and the traffic demand as well. We compute the load factor for each station, and finally we estimate the SINR which depends on the RSRQ. As a second step we assign again the test point to the base station on the basis of the best SINR. From there 
we estimate again the load factor for each station. This second step is repeated several times (10 times) to try to reach a stable network configuration.

The collected traffic data come from a real UMTS network. The tests consider three different scenarios originating from the traffic of one day, as shown in Figure 3. Three scenarios were selected at different times of the day as follows: a first scenario at $8 \mathrm{am}$ with low traffic and 482 users dropped randomly in the network; a second scenario at $3 \mathrm{pm}$ with medium traffic and 1,019 users; and a third scenario at $6 \mathrm{pm}$ with high traffic and 1,471 users. We are considering that all users are accessing the network at the same time (saturated traffic condition).

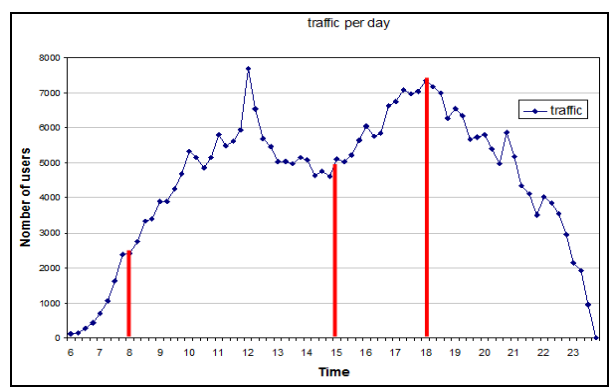

Figure 3. Example of day traffic with three chosen scenarios

Figure 4 shows the concentration of the traffic in the $3 \mathrm{rd}$ scenario at $6 \mathrm{pm}$ (high traffic). The traffic is represented by a color gradient. The light color shows a low traffic, while the dark color shows higher traffic.

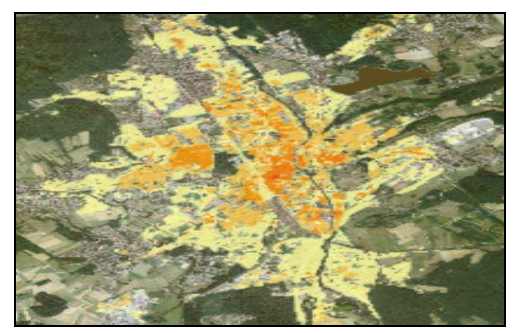

Figure 4. Example of traffic concentration at $6 \mathrm{pm}$

\subsection{Interference model results}

To compare the performances of both reuse $1 \times 3 \times 1$ and reuse $1 \times 3 \times 3$ patterns, the number of users in outage is used as a performance metric. The minimum required SINR is equal to 0.9 $\mathrm{dB}[8]$. Below this value of SINR, one user cannot establish a communication as defined in Equation 8:

$$
\gamma_{\mathrm{b}, \mathrm{t}, \mathrm{s}}=\frac{\mathrm{p}_{\mathrm{b}, \mathrm{t}}^{\mathrm{R}} \mathrm{f}_{\mathrm{b}}}{\sum_{\mathrm{b}^{\prime} \neq \mathrm{b}, \mathrm{f}_{\mathrm{b}}=\mathrm{f}_{\mathrm{b}^{\prime}}} \mathrm{p}_{\mathrm{b}^{\prime}, \mathrm{t}}^{\mathrm{R}} \mathrm{f}_{\mathrm{b}^{\prime}} \delta_{\mathrm{b}, \mathrm{s}} \delta_{\mathrm{b}^{\prime}, \mathrm{s}}+\mathrm{n}_{0} \mathrm{w}}<\gamma^{\mathrm{MIN}}
$$

where $\gamma^{\mathrm{MIN}}$ is the required threshold.

Figure 5 depicts the number of users in outage obtained with both reuses pattern: scheme $1 \times 3 \times 1$ (abbreviated scheme 1 in Figure 5) and scheme $1 \times 3 \times 3$ or simply scheme 3 (Figure 5 ). In the network design we test for scheme $1 \times 3 \times 3$, the sub-band assignment depends on the azimuth orientation of the sectors. Sectors in opposite direction from neighbor sites are assigned different frequency groups. The program implementing our model is developed in $\mathrm{C}++$. We run the program ten times at least to get stable results (as there is a random process in the system computation); an example of three scenarios of traffic is presented in the following (Figure 5) to show the interest of using the reuse scheme $1 \times 3 \times 3$ in a real network design and traffic load.

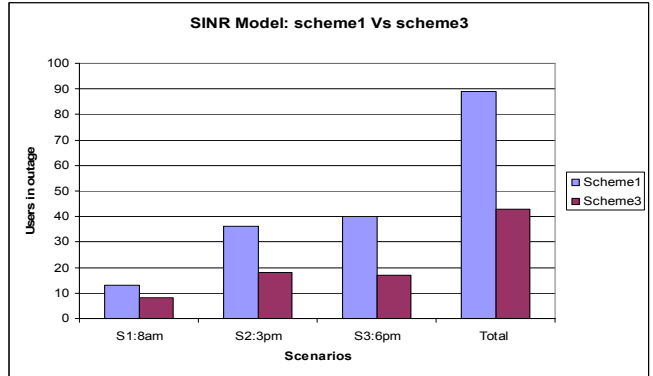

Figure 5. Reuse scheme 1and scheme 3 results with 3 scenarios

We remind that the frequency reuse 1 (or $1 \times 3 \times 1$ ) scheme whereby the whole available bandwidth is used in each cell/sector and the frequency reuse 3 (also called $1 \times 3 \times 3$ ) scheme in which the entire bandwidth is divided into 3 non-overlapping groups and assigned to 3 co-site sectors within each cell.

In Figure 5 we see that the number of users in outage is more important in scheme $1 \times 3 \times 1(13,36$, and 40$)$ than in scheme $1 \times 3 \times 3$ $(8,18$ and 17) for the three scenarios of traffic load considered here ( $8 \mathrm{am}, 3 \mathrm{pm}$ and $6 \mathrm{pm}$ respectively). These results are consistent since the total bandwidth used in pattern 1 can assign more resources, but would jeopardize the rate offered by the network. This is due mainly to the undesirable inter-cell interference generated by neighboring cells using the same frequency set. Such a dense reuse is an obvious pitfall which limits the throughput at the cell edge. We can also note according to these results that, the higher the traffic, the higher the number of outage users, whatever the frequency pattern solution.

\subsection{Frequency and tilt optimization}

The second part of this study is dedicated to robust optimization. It shows that optimizing a number of network configuration parameters like antenna frequency and tilt orientation helps considerably to meet variant of performance requirements. A greedy algorithm is used to examine how the solution of the antenna configuration, behaves under realistic scenarios.

\subsubsection{Results with non robust and robust optimization}

\subsubsection{Frequency optimization results}

The results of optimization are shown in the Figures 6 and 7. We emphasize that for the non robust (each scenario tackled alone) and for the robust optimization (all scenarios together) we use the same algorithm but in case of robustness the evaluation function is the Equation 7 and takes into account the configuration of the frequency considering all the scenarios simultaneously. It means that, for each frequency of the network, we evaluate the noncovered users in the three scenarios. So, we run the same algorithm 4 times (one run for each optimization), we use the evaluation function Equation 6 to optimize the 3 scenarios separately; then we use the evaluation function (Equation 7) for the robust optimization using the 3 scenarios at the same time. 


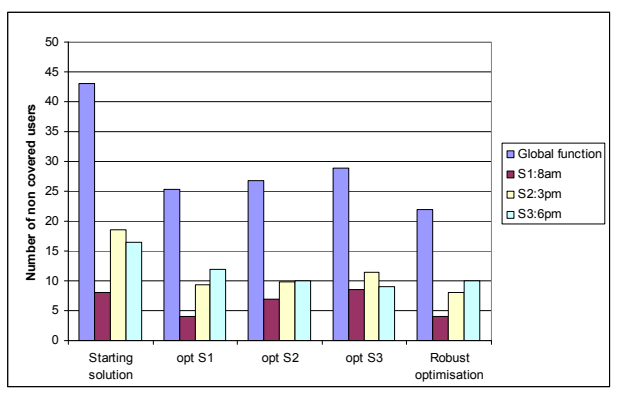

Figure 6. Three scenarios of robust optimization interest with deterministic frequency allocation

The $\mathrm{x}$-axis represents the starting solution and the optimization of scenarios $s_{1}, s_{2}$ and $s_{3}$ separately and the robust optimization at the end. The y-axis shows the number of users in outage for each scenario $s_{1}, s_{2}, s_{3}$ and total number. We can note that scenariol optimization has the smallest number of non-covered users when evaluating $s_{I}$ (4 users) comparing to the other cases $(8,7,9)$. The same analysis can be done for the scenario $s_{3}$ and it is different for $s_{2}$ but not far away from the best one. After $20 \mathrm{mn}$ of run there is no guarantee on the solution quality. We observe that the result of the robust optimization is a tradeoff between the three scenarios, the best for $s_{1}$ and $s_{2}$ but not the best for s3. Finally, the fitness function value of non-covered users for all cases corresponds to the global best solution (blue color in the right part), while in other situations, starting solution and non robust cases, the global function values are 43, 25, 27 and 29 respectively from left to right part of the Figure 6. The robust optimization does a better compromise between all scenarios. This result shows how the robust approach is important for the remaining of this study. Different variants of the algorithm have been tested by varying several parameters. We run the program 20 minutes for each optimization in Test 2 and Test 3, and keep the best solution for the considered fitness function.

Test 1 (Figure 6): the initial frequency plan is deterministically assigned and the sites are processed respecting their rank in the input file.

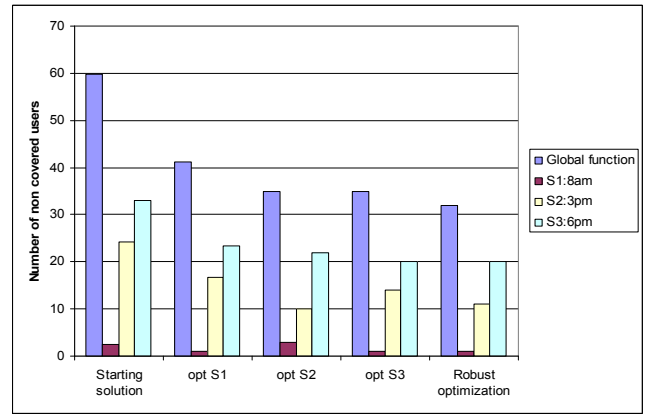

Figure 7. Three scenarios of robust optimization with random frequency allocation

Test 2: the initial frequency plan is deterministically assigned and the sites are randomly processed during optimization. The results are similar to the test 1 so we do not plot it.

Test 3 (Figure 7): the initial frequency plan is randomly assigned to the co-site sectors and the sites are randomly processed during optimization.

\subsubsection{Tilt optimization results}

Robust optimization results for the tilt parameter are highlighted in the right part of each test (Figure 8 and 9). Several tests have been made to show the tilt parameter effect on the number of non covered users, we limit at showing 2 tests varying the neighborhood sites process and the stopping criteria.

Test 1 (Figure 8): the algorithm presented on 4.2 is implemented. It means that with the same starting solution ( 3 scenarios of traffic and the operating tilts in real network), the site neighborhood search is done according their storage in the data file. In addition of sites search, the algorithm stops once there is no improvement of the solution. Results are shown in Figure 8.

Figure 8 shows 5 parts, from the right to left: starting solution, optimization of scenarios $s_{1}, s_{2}$ and $s_{3}$ separately, and then robust optimization. The starting point of the algorithm is the starting solution $(43,8,18$ and 17) which represents the number non covered users in the global function (Equation 7) and scenario function (Equation 6) for each scenario.

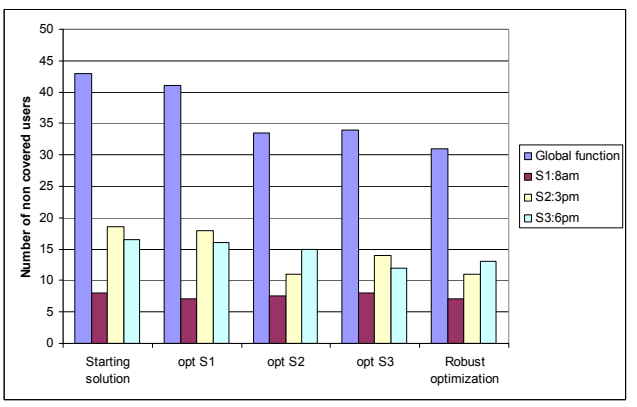

Figure 8. Three scenarios of robust optimization with deterministic tilt configuration

We emphasize that the optimization of each scenario provides better results when each scenario is tackled separately $(7,11,12)$ respectively, but impacts the global function. In robust optimization, we can easily note that the configuration found by the algorithm is a better compromise between all scenarios, $\mathrm{f}^{\text {Rob }}=31$ compared with starting solution and the scenarios optimization (43, 41, 33 and 34).

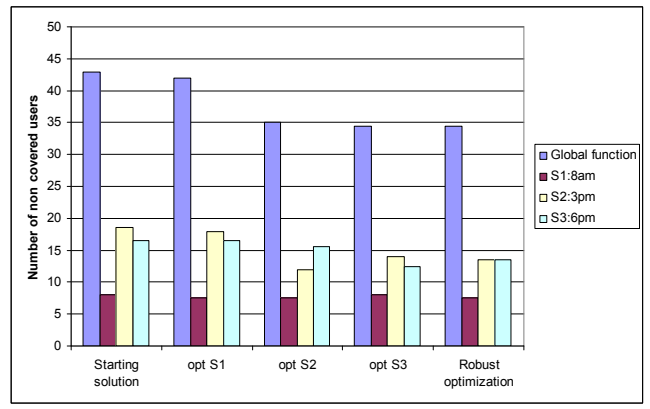

Figure 9. Three scenarios of robust optimization with random tilt configuration

Test 2 (Figure 9): we keep the same initial conditions; but vary the sites neighborhood search (random search) and stopping criteria (an hour of run). It shows the same results as in Figure 8. It confirms that optimizing by means of robust function can improve the network coverage with regard to traffic distribution. 
The Figure 10 represents à comparison between the 2 parameter setting optimizations; with the same initial condition (Test 1 in the frequency case and Test 1 in the tilt optimization case).

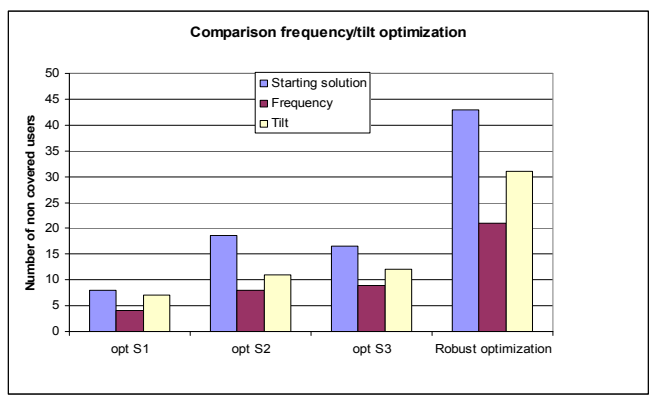

Figure 10. Comparison between frequency and tilt optimization: Sites processed according their storage in data file; stopping criteria when there is no improvement

According to these results, we note that optimizing the frequency parameter provides better results while taking coverage as a performance indicator: less uncovered user for $s_{1}$ case $(4<7) ; s_{2}$ $(8<11)$ and $s_{3}(9<12)$ and also in robust optimization $(21<31)$. Such results are expected to happen. This is due mainly to the undesirable inter-cell interference generated by neighboring cells using the same frequency set. It impacts more the SINR quality comparing to the tilt parameter.

\section{CONCLUSION}

In this paper we have shown the interference model and the interest of robust approach with respect to traffic distribution in LTE downlink system. The analysis has been carried out using model radiation pattern and simple model of system performance. The interference model has been validated based on SINR computation and comparing two reuse schemes $(1 \times 3 \times 1$ and $1 \times 3 \times 3)$ under realistic scenarios. With respect to coverage, it has been observed that the reference reuse $1 \times 3 \times 3$ presents best results with respect to the number of covered users, independently from the traffic demand. It shows also that the load factor could impact the quality of signal at end users. It is an important indicator because it highlights the overloaded cells which represent the bottleneck of the network. Using system simulations, we studied how the frequency and the tilt parameters setting affect the coverage of the macro-cellular scenario. Different combinations of frequencies and tilts are used and obtained results show how coverage indicator is sensitive to the combination, and also to the traffic inaccuracies. Simple algorithms used here confirm the interest of robust approach irrespective of realistic traffic load. As perspectives, we aim in further studies at analyzing the influence of the load factor throughout the capacity performance metric to bring out the overloaded cells which represent the bottlenecks of the network. We will also study the impact of the power transmission parameter on the interference model and other performance metrics (throughput, capacity). Furthermore, robust optimization approaches like the Variable Neighborhood Search and Tabu Search will be studied to highlight the impact of the traffic uncertainty in the deployment of the network.

\section{REFERENCES}

[1] Mao, M., Maaref, A. and Teo, K. 2008. Adaptive soft frequency reuse for inter-cell interference coordination in SC-FDMA based 3GPP LTE uplinks," Proc. IEEE Global Telecommunications Conference (GlobeCom), New Orleans LO, USA.
[2] Rahman, M. and Yanikomeroglu, H. 2010. Enhancing cell edge performance: A downlink dynamic interference avoidance scheme with inter-cell coordination," IEEE Transaction on Wireless Telecommunication, vol.9, no.4, pp. 1414-1425.

[3] Rahman, M., and Yanikomeroglu, H. 2009. Interference Avoidance With Dynamic Inter-Cell Coordination for Downlink LTE System. Proc. IEEE Wireless Communication and Networking Conference (WCNC).

[4] 3GPP R1-050738. 2005. Interference mitigationConsiderations and results on frequency reuse, Siemens.

[5] 3GPP R1-050507. 2005. Soft Frequency Reuse Scheme for UTRAN LTE," Huawei.

[6] Yilmaz, O.N.C, Hamalainen, S. and Hamalainen, J. 2009. System level analysis of vertical sectorisation for 3GPP LTE. Proc. IEEE 6th International Symposium On Wireless Ccommunication System (CSWCS ).

[7] Siomina, I., Varbrand, P. and Yuan, D. 2006. Automated optimization of service coverage and base station antenna configuration in UMTS networks. IEEE Wireless Communications. Vol 13. no. 6. pp. 16-25.

[8] Schoenen, R., Zirwas, W., and Walke, B.H. 2008. Capacity and coverage analysis of a 3 GPP-LTE multihop deployment scenario. Proc. IEEE International Conference On Communications Workshops.

[9] Holma, H., and Toskala, T. 2009. LTE for UMTS OFDMA and SC-FDMA Based Radio Access, John Wiley \& sons Ltd Edition.

[10] Yilmaz, O.N.C and Hamalainen, S. 2009. Comparaison of remote electrical and mechanical antenna downtilt performance for 3GPP LTE. Proc. IEEE 70th Vehicular Conference Fall (VTC-2009 FALL).

[11] Gondran, A., Baala, O., Caminada, A., Mabed, M. 2008. Interference management in IEEE 802.11 frequency assignment. Proc. IEEE Vehicular Technology Conference (VTC Spring), pp. 2238-2242, Singapore.

[12] Mabed, H., Caminada, A.. 2006. Geometric criteria to improve the interference performances of cellular network", Proc. 64th IEEE Vehicular Technology Conference (VTC Fall), pp. 1-5, Montreal.

[13] Altman, Z., Picard, J.M., Ben Jamaa, S., Fourestie, B., Caminada, A., Dony, T., Morlier, J.F., Mourniac, S. 2002. New challenges in automatic cell planning of UMTS networks", Proc. 56th IEEE Vehicular Technology Conference (VTC Fall), pp. 951-954.

[14] Didan, I., and Kurochkin, A. 2006. The impacts of antenna azimuth and tilt installation accuracy on UMTS Network Performance, Bechtel Corporation.

[15] Athley, F. and Johansson, M. 2010. Impact of Electrical and Mechanical Antenna Tilt on LTE Downlink System Performance. IEEE Vehicular technology conference, Ericsson Res., Ericsson AB, Göteborg, Sweden.

[16] Hata, M.1980. Empirical formula for propagation loss in land mobile radio services. In: IEEE Transactions on Vehicular Technology, vol.29, No. 3. 